\title{
Amiodarone for Postoperative Atrial Fibrillation in Lung Cancer Patients: A 6-Year Follow-Up Study
}

\author{
Cecilie Enevoldsen1,2, Britt Borregaard1,2, Sara Schødt Riber ${ }^{1,2}$, Lars Peter Schødt Riber 1,2* \\ ${ }^{1}$ Department of Cardiothoracic and Vascular Surgery, Odense University Hospital, Odense, Denmark \\ ${ }^{2}$ Faculty of Health Sciences, University of Southern Denmark, Odense, Denmark \\ Email: ${ }^{*}$ lars.riber@rsyd.dk
}

How to cite this paper: Enevoldsen, C., Borregaard, B., Riber, S.S. and Riber, L.P.S. (2020) Amiodarone for Postoperative Atrial Fibrillation in Lung Cancer Patients: A 6-Year Follow-Up Study. Open Journal of Thoracic Surgery, 10, 6-18.

https://doi.org/10.4236/ojts.2020.101002

Received: December 13, 2019

Accepted: January 28, 2020

Published: January 31, 2020

Copyright $\odot 2020$ by author(s) and Scientific Research Publishing Inc. This work is licensed under the Creative Commons Attribution International License (CC BY 4.0).

http://creativecommons.org/licenses/by/4.0/

cC (i) Open Access

\begin{abstract}
Background: The preventive effect of prophylactic amiodarone on postoperative atrial fibrillation (POAF) in patients undergoing thoracic surgery has previously been demonstrated. Meanwhile, the long-term effect remains unknown. We investigated the long-term effect of prophylactic amiodarone, in patients undergoing surgery for lung cancer, along with the long-term prognosis of patients with POAF within 6 years of follow-up. Methods: Using data from national databases, we retrospectively analysed 250 patients included and randomized, in the randomized control trial, PASCART, in which patients were allocated to receive either amiodarone or placebo as prophylaxis for POAF. Prophylactic groups, and subgroups, were compared on a number of outcomes. Long-term overall survival was evaluated using Kaplan-Meier survival curves, and Cox proportional hazards models were used for multivariable analysis. Competing risk analysis was used to evaluate time-to-event data in presence of competing risk. Results: When comparing the prophylactic groups, prophylaxis with amiodarone was not convincingly related to a higher frequency of long-term side effects. Patients who developed POAF, were more likely to develop late atrial fibrillation (AF) and POAF was associated with an increased risk of late $\mathrm{AF}$ in both the competing risk analysis hazard rate (HR) 4.80 [95\% 1.75; 13.18] and multivariable analysis of the Cox regression, HR 5.03 [95\% 1.80; 14.10]. When comparing the Kaplan Meier survival curves between groups, we found no statistically significant difference in the long-term overall survival. Conclusions: Intravenous prophylactic amiodarone is safe in patients undergoing lung cancer surgery. POAF is associated with an increased risk of late AF.
\end{abstract}

\section{Keywords}

Lung Cancer, Atrial Fibrillation, Postoperative Care, Pharmacology 


\section{Introduction}

Since 1943, when the first supraventricular arrhythmia following pulmonary resection was reported, postoperative atrial fibrillation (POAF) has been a common and well-recognized complication to non-cardiac thoracic surgery [1] [2] [3]. The incidence is ranging from $3 \%-60 \%$, with a tendency towards an even higher rate, in patients undergoing more invasive surgical procedures, such as lobectomy and pneumonectomy [2] [3] [4] [5]. While atrial fibrillation is usually considered to be an unhazardous and transient arrhythmia, POAF is associated with a poorer prognosis regarding morbidity and mortality [3] [4]. The etiology and pathophysiological interactions, which leads to the development of POAF, remains unknown, although, studies have shown a higher risk correlating with age, male sex, obesity, hypertension, extent of surgery, prior atrial fibrillation (AF) or arrhythmia, history of heart disease, chronic obstructive pulmonary disease (COPD) and brain natriuretic peptide (BNP) levels [2] [3] [4] [6].

Considering the potentially negative impact of POAF on morbidity and mortality, possibly preventive effect of antiarrhythmic drugs has been investigated [7] [8] [9] [10] [11]. Between 2008 and 2011, Riber et al. conducted the randomized control trial, PASCART, of which 242 patients undergoing surgery for lung cancer were randomized to receive either amiodarone or placebo, as prophylaxis for POAF. Follow-up was 30 days after surgery [12]. When comparing the groups, patients in the intervention group, experienced a significant reduction in the risk of atrial fibrillation; $23 \%$ with a number needed to treat at 4.4 [12]. Amiodarone is known as one of the most effective anti-arrhythmic drugs, but due to adverse short-term effects when administered continually and unknown long-term effects, the use has so far been recommended with caution [13].

Thus, the objectives of this follow-up study among patients receiving prophylactic amiodarone and placebo are to: 1) describe differences in baseline characteristics, the frequency of stroke and heart failure, readmission, late $\mathrm{AF}$, long-term all-cause mortality, and long-term side effect; 2) investigate associations between amiodarone and placebo and the risk of readmission, developing late $\mathrm{AF}$ and long-term all-cause mortality. And furthermore, among patients developing POAF to: 3) describe differences in baseline characteristics, the frequency of stroke and heart failure, readmission, late AF and long-term all-cause mortality, and to, 4) investigate the associations between POAF and the risk of readmission, late AF and long-term all-cause mortality.

\section{Patients and Methods}

\subsection{Study Population and Data Extraction}

Following the approval of the Danish Data Protection Agency and the Danish Patient Safety Authority, we conducted a retrospective, register-based 6-year follow-up study on the population of 250 patients, included in the randomized control trial, PASCART, at the Department of Cardiothoracic and Vascular Surgery, Aarhus University Hospital [12]. Follow-up data were collected from 
the Danish Register of Causes of Death and the Danish National Patient Registry [14] [15] [16]. Baseline characteristics, including patient characteristics, relevant comorbidities, peri- and post-operative data were extracted from the original dataset.

Eligible subjects were identified for inclusion and exclusion, based on the criteria, listed in the original study. Inclusion criteria were; 1 ) elective lobectomy or pneumonectomy; 2) age above 18 years; 3) willingness to participate; and 4) provision of informed consent. Exclusion criteria were: a) previous heart or lung surgery; b) resting heart rate below 40 beats per minute; c) hypotension with systolic blood pressure below $80 \mathrm{~mm} \mathrm{Hg}$; d) atrial-ventricular blockage of any degree or sick sinus syndrome; e) preoperative AF or flutter; f) known previous AF or flutter lasting more than 1 month; g) hepatic dysfunction (alanine aminotransferase level more than twice the upper normal limit); h) hyperthyroidism; i) pregnancy; $j$ ) breastfeeding; $k$ ) in treatment with monoamine oxidase inhibitors (MAOI); l) QTc interval longer than $440 \mathrm{~ms}$ for men and $460 \mathrm{~ms}$ for women; or m) known adverse reactions to amiodarone [12]. Additionally, patients were excluded in this study due to protocol violation (Figure 1).

\subsection{Follow-Up Data and Definitions}

Heart failure, readmission, late AF, readmission and stroke, which will be referred

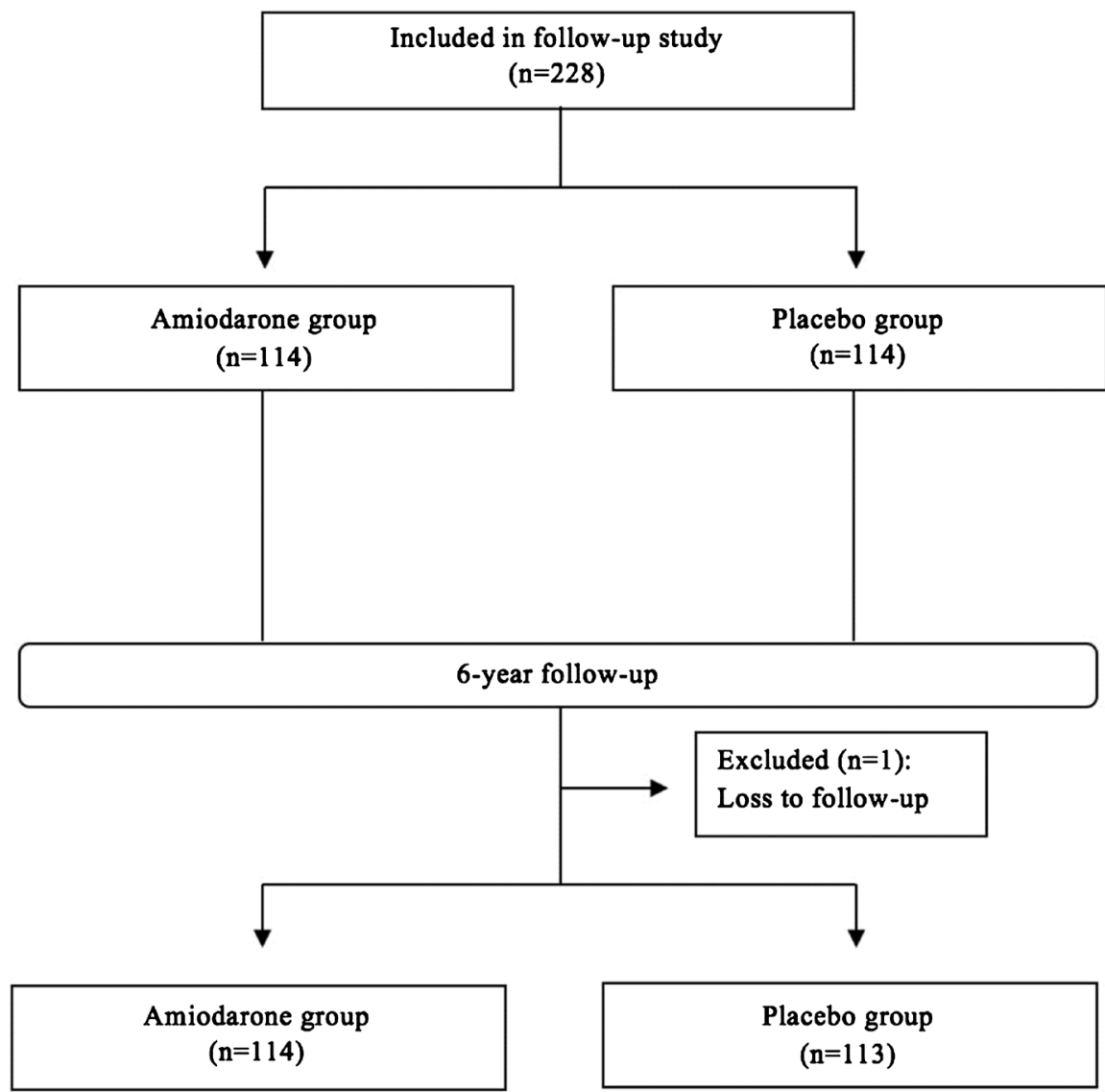

Figure 1. Flowchart. 
to as HARS (Heart failure, late Atrial fibrillation, Readmission and Stroke), long-term side effects, long-term all-cause mortality and mortality due to cardiovascular disease, were collected as follow-up data. Diagnostic codes, classified by the ICD-10 classification, were used to defined and create binary variables for follow-up data, including heart failure, late AF, and stroke, long-term side effects, long-term all-cause mortality and mortality due to cardiovascular disease. When evaluating long-term side effects, liver, lung, thyroid, and skin diagnosis reflecting known amiodarone side-effects, were studied in particular. Before creating variables, diagnostic codes, coding for the same disease or organ system e.g. "neurological disease", were placed into the same categories. In each category, diagnostic codes were further divided into subcategories e.g. "neuropathy". Diagnostic codes were excluded if they were uncategorizable, and only occurred once. Diagnostic codes for AF, atrial flutter and unspecified atrial flutter or fibrillation, were all used to define the variable of late AF. Late AF and readmission were evaluated on time to the first event, defined as the interval from the surgery date, to the first AF and readmission respectively, in the follow-up period. Furthermore, readmission was evaluated by the number of patients readmitted during the follow-up period. Long-term mortality was evaluated by all-cause mortality, and cardiovascular mortality. All-cause mortality was defined as death of any cause, and cardiovascular mortality defined as death due to cardiovascular diseases, during the follow-up period.

\subsection{Statistical Methods}

Prophylactic groups (intervention group and placebo group) and sub-groups (patients who developed POAF, and patients who stayed in sinus rhythm) were analysed separately.

Differences among groups, including differences in baseline characteristics and differences in stroke, heart failure, readmission, late AF, long-term all-cause mortality and long-term side effects among groups were tested using Pearson's $\chi^{2}$ test of independence or Fisher's exact test, for categorical variables, and Students t-test or Mann-Whitney $U$ test, for numerical variables. Numerical variables were presented as median and quartiles (interquartile range (IQR) from 25 th to 75 th quartile) or mean and standard deviation (SD), depending on the normality of distribution, tested by the Shapiro-Wilk test. Categorical variables were reported by the number and percentage of subjects in each category.

The association between 1) amiodarone and readmission, late $\mathrm{AF}$, and long-term all-cause mortality 2) POAF and readmission, late AF, and long-term all-cause mortality, were evaluated by estimating the hazard ratio, using Cox multivariable proportional hazard analysis, adjust for potential confounders. Results were presented as hazard ratios (HR) with 95\% confidence intervals (CI).

Long-term mortality rates for prophylactic groups and subgroups during the follow-up period were estimated by the use of Kaplan-Meier survival curves. For 
patients surviving the follow-up, the follow-up time was 6 years. For patients dying within 6 years the time-to-death was calculated from the date of surgery to date of death. Log-rank test was used to compare mortality rates between prophylactic groups and subgroups.

Time to first AF and readmission was calculated from the date of surgery to date of first $\mathrm{AF}$ and readmission respectively, and compared within prophylactic groups and subgroups. In the presence of competing events, the time to event data, were analysed using the cumulative incidence function (CIF) in an univariable Fine and Gray Proportional Hazard Model [17].

Multiple imputations were not performed in case of missing data. All p-values are two-tailed, and a p-value $<0.05$ was considered as statistically significant. All the data were analysed using SPSS Statistics 25 SPSS (IBM Corp., Armonk, NY, USA), Stata Statistical Software 15.0 (Stata Corp LLC, College Station, TX).

\section{Results}

In this current study, we conducted a 6-year follow-up on patients included in the randomized control trial, PASCART. Between 2008 and 2011, 386 patients were enrolled for lobectomy or pneumonectomy at Aarhus University Hospital, Denmark. In total, 228 of those patients were eligible and included in this follow-up. One of the patients included was lost to follow-up. Therefore, 114 in the amiodarone group, and 113 in the placebo group, were included in this long-term follow-up study (Figure 1).

\subsection{Baseline Results}

There was no statistically significant difference between treatment groups in baseline characteristics presented in Table 1, except the incidence of POAF, which were higher in the placebo group (Table 1). None of the patients had any history of arrhythmia, AF or atrial flutter. Moreover, none of the patients had undergone any heart or lung surgery. When comparing the subgroups, patients who developed POAF were more likely to have hypertension (Table 1). None of the patients had any postoperative, cerebral insult, acute myocardial infarction, blockage or arrhythmia, besides POAF.

\subsection{Long-Term Side Effects}

As described in the method section, potentially long-term side effects were evaluated based on diagnose codes. When comparing the subcategories, there were no statistically significant differences between the amiodarone group and the placebo group, except a difference in the incidence of head and neck cancer ( $p=0.029$, with $5(4 \%)$ cases in the placebo group, and none in the amiodarone group. Uncategorized diagnostic codes were compared as well, and only a statistically significant difference, in the incidence of the diagnose code, DJ869: Pleural empyema UNS ( $p=0.013$ ) was found, with $6(5 \%)$ cases in the placebo group, and none in the amiodarone group. 
Table 1. Baseline characteristics.

\begin{tabular}{|c|c|c|c|c|c|c|c|}
\hline & \multirow{2}{*}{ All } & \multicolumn{2}{|c|}{ Prophylactic Group } & \multirow{2}{*}{$\mathrm{p}^{*}$} & \multicolumn{2}{|c|}{ Subgroup } & \multirow{2}{*}{$\mathrm{p}^{*}$} \\
\hline & & Amiodarone & Placebo & & POAF & NO POAF & \\
\hline \multicolumn{8}{|l|}{ Characteristics } \\
\hline $\mathrm{N}(\%)$ & $228(100)$ & $114(50)$ & $114(50)$ & N/A & $47(21)$ & $181(79)$ & N/A \\
\hline Age (mean, SD) & $66(59 ; 72)$ & $65(59 ; 71)$ & $66(59 ; 74)$ & 0.405 & $68(62 ; 74)$ & $64(58 ; 71)$ & 0.081 \\
\hline Sex (male, n (\%)) & $117(51)$ & $57(50)$ & $60(52)$ & 0.691 & $27(57)$ & $90(50)$ & 0.345 \\
\hline Current smoker (n (\%)) & $86(38)$ & $39(34)$ & $47(41)$ & 0.274 & $17(36)$ & $69(38)$ & 0.806 \\
\hline Diabetes mellitus (n (\%)) & $21(9)$ & $14(12)$ & $7(6)$ & 0.109 & $4(9)$ & $17(9)$ & 1.000 \\
\hline Hypertension (n (\%)) & $75(33)$ & $43(38)$ & $32(28)$ & 0.121 & $22(47)$ & $53(29)$ & $0.023^{*}$ \\
\hline Hypercholesterolemia (n (\%)) & $34(15)$ & $19(17)$ & $15(13)$ & 0.457 & $8(17)$ & $26(14)$ & 0.649 \\
\hline COPD (n (\%)) & $23(10)$ & $13(11)$ & $10(9)$ & 0.509 & $5(11)$ & $18(10)$ & 1.000 \\
\hline Previous MI (n (\%)) & $4(2)$ & $* *$ & $* *$ & 1.000 & $* *$ & $* *$ & 1.000 \\
\hline Preoperative use of $\beta$-blockers (n (\%)) & $29(13)$ & $16(14)$ & $13(11)$ & 0.551 & $7(15)$ & $22(12)$ & 0.616 \\
\hline Creatinine $(\mu \mathrm{mol} / \mathrm{L})$ (median, IQR) & $66(57 ; 77)$ & $66(58 ; 77)$ & $65(54 ; 76)$ & 0.180 & $68(60 ; 78)$ & $65(55 ; 76)$ & 0.081 \\
\hline \multicolumn{8}{|l|}{ Peri- and Postoperative Characteristics } \\
\hline Postoperative atrial fibrillation (n (\%)) & $47(21)$ & $10(9)$ & $37(33)$ & $<0.001^{\star}$ & $47(21)$ & $181(79)$ & N/A \\
\hline Postoperative arrhythmia (n (\%)) & $* *$ & ** & $* *$ & N/A & $* *$ & $* *$ & N/A \\
\hline Postoperative blockage (n (\%)) & $* *$ & $* *$ & $* *$ & $\mathrm{~N} / \mathrm{A}$ & $* *$ & $* *$ & N/A \\
\hline Reoperation (n (\%)) & $8(4)$ & $5(5)$ & $* *$ & 0.499 & $* *$ & $6(3)$ & 0.668 \\
\hline Right side lobectomy (n (\%)) & $120(53)$ & $59(52)$ & $61(54)$ & 0.791 & $26(55)$ & $94(52)$ & 0.679 \\
\hline Bilobectomy (n (\%)) & $5(2)$ & $* *$ & $* *$ & 1.000 & ** & $* *$ & 0.061 \\
\hline Pneumonectomy (n (\%)) & $8(4)$ & $4(4)$ & $4(4)$ & 1.000 & $* *$ & $6(3)$ & 0.670 \\
\hline \multicolumn{8}{|l|}{ Tumour stage (n (\%)) } \\
\hline Tumour I & $87(39)$ & $39(35)$ & $48(43)$ & 0.260 & $19(40)$ & $68(38)$ & 0.802 \\
\hline Tumour II & $99(44)$ & $56(51)$ & $43(38)$ & 0.062 & $23(49)$ & $76(43)$ & 0.462 \\
\hline Tumour III & $31(14)$ & $13(12)$ & $18(16)$ & 0.361 & $* *$ & $28(16)$ & 0.096 \\
\hline Tumour IV & $7(3)$ & $* *$ & $4(4)$ & 1.000 & $* *$ & $5(3)$ & 0.639 \\
\hline \multicolumn{8}{|l|}{ Nodulus stage (n (\%)) } \\
\hline Nodulus I & $32(14)$ & $15(14)$ & $17(15)$ & 0.743 & $6(13)$ & $26(15)$ & 0.738 \\
\hline Nodulus II & $36(16)$ & $14(13)$ & $22(20)$ & 0.162 & $7(15)$ & $29(16)$ & 0.805 \\
\hline \multicolumn{8}{|l|}{ Metastasis stage (n (\%)) } \\
\hline Metastasis I & $10(5)$ & $5(5)$ & $5(4)$ & 1.000 & $* *$ & $9(5)$ & 0.692 \\
\hline Metastasis II & ** & $* *$ & $* *$ & N/A & $* *$ & $* *$ & N/A \\
\hline Tumour size (mm) (median, IQR) & $31(20 ; 50)$ & $32(20 ; 50)$ & $30(20 ; 45)$ & 0.671 & $30(20 ; 41)$ & $32(20 ; 50)$ & 0.669 \\
\hline Length of stay in ICU (days) (median, IQR) & $1(1 ; 1)$ & $1(1 ; 1)$ & $1(1 ; 1)$ & 0.774 & $1(1 ; 1)$ & $1(1 ; 1)$ & 0.785 \\
\hline $\begin{array}{l}\text { Length of stay in referring department (days) } \\
\text { (median, IQR) }\end{array}$ & $4(3 ; 5)$ & $4(3 ; 5)$ & $4(3 ; 5)$ & 0.699 & $4(3 ; 5)$ & $4(3 ; 5)$ & 0.334 \\
\hline
\end{tabular}

$\mathrm{COPD}=$ Chronic obstructive pulmonary disease. $\mathrm{MI}=$ myocardial infarction. $\mathrm{IQR}=$ Interquartile range. $\mathrm{ICU}=$ Intensive Care Unit. ${ }^{*}$ Significant $p$-values; **Three or fewer cases. 


\subsection{HARS and Long-Term Mortality}

When comparing the prophylactic groups, there was no statistically significant difference in HARS (Table 2). When comparing the subgroups, there was a statistically significant difference in late AF, which was more frequent among patients who developed POAF. No statically significant difference was found in readmission, stroke and heart failure between subgroups. Of the 227 patients included in the follow-up analysis, $44 \%$ died during the follow-up. Only $4 \%$ died of the cardiovascular diseases, while $94 \%$ died from other causes (Table 2). Cause of death was not registered in two patients (2\%). When comparing the percentage of deaths between groups, no statically significant difference was found.

\subsection{Risk Analysis}

In the univariable and multivariable analysis, amiodarone was not associated with an increased risk of readmission, late AF or long-term all-cause mortality (Table 3 \& Figure 2). POAF was associated with an increased risk of late AF in both the univariable HR 4.80 [95\% 1.74; 13.24] and multivariable analysis, HR 5.03 [95\% 1.80; 14.10] (Table 4). In the univariable and multivariable analysis, POAF was not associated with an increased risk of readmission and long-term all-cause mortality (Table 4 \& Figure 2).

\subsection{Long-Term Survival}

In both the prophylactic groups and subgroups, the survival during the follow-up

Table 2. Descriptive follow-up data on HARS and mortality.

\begin{tabular}{|c|c|c|c|c|c|c|c|}
\hline & \multirow{2}{*}{ All } & \multicolumn{2}{|c|}{ Prophylactic Group } & \multirow{2}{*}{$\mathrm{p}^{*}$} & \multicolumn{2}{|c|}{ Subgroup } & \multirow{2}{*}{$\mathrm{p}^{*}$} \\
\hline & & Amiodarone & Placebo & & POAF & NO POAF & \\
\hline \multicolumn{8}{|l|}{ Variables } \\
\hline All-cause mortality (n (\%)) & $100(44)$ & $49(43)$ & $51(45)$ & 0.745 & $21(46)$ & $79(44)$ & 0.807 \\
\hline Readmission (n (\%)) & $203(89)$ & $101(89)$ & $102(90)$ & 0.540 & $42(91)$ & $161(89)$ & 0.792 \\
\hline Late AF (n (\%)) & $15(7)$ & $5(4)$ & $10(9)$ & 0.176 & $8(17)$ & $7(4)$ & $0.004^{\star}$ \\
\hline Stroke (n (\%)) & $10(4)$ & $4(4)$ & $6(5)$ & 0.539 & ** & $7(4)$ & 0.428 \\
\hline Heart failure (n (\%)) & $* *$ & $* *$ & ** & 0.498 & $* *$ & $* *$ & 1.000 \\
\hline
\end{tabular}

${ }^{*}$ Significant $p$-values; ${ }^{*}$ Three or fewer cases.

Table 3. Unadjusted and adjusted Cox Proportional Hazard Regression analysis of the association between amiodarone and readmission, Late AF and all-cause mortality.

\begin{tabular}{ccccccc}
\hline & \multicolumn{2}{c}{ Readmission } & \multicolumn{2}{c}{ Late AF } & \multicolumn{2}{c}{ All-Cause Mortality } \\
\cline { 2 - 7 } & Univariable & Multivariable & Univariable & Multivariable $^{\mathrm{b}}$ & Univariable & Multivariable $^{\mathrm{a}}$ \\
\cline { 2 - 7 } & HR $(95 \% \mathrm{CI})$ & HR $(95 \% \mathrm{CI})$ & HR $(95 \% \mathrm{CI})$ & HR $(95 \% \mathrm{CI})$ & HR $(95 \% \mathrm{CI})$ & HR $(95 \% \mathrm{CI})$ \\
\hline \multirow{2}{*}{ Amiodarone } & $0.93(0.70 ; 1.22)$ & $0.95(0.71 ; 1.27)$ & $0.49(0.17 ; 1.42)$ & $0.77(0.25 ; 2.41)$ & $0.94(0.64 ; 1.39)$ & $0.96(0.64 ; 1.43)$ \\
\hline
\end{tabular}

${ }^{\mathrm{a}}$ Adjusted for age, sex, and POAF, ${ }^{\mathrm{b}}$ Adjusted for POAF, POAF $=$ postoperative atrial fibrillation. Late AF = Atrial fibrillation developing after 30 postoperative days. 
Table 4. Unadjusted and adjusted Cox Proportional Hazard Regression analysis of the association between POAF and readmission, Late AF and all-cause mortality.

\begin{tabular}{ccccccc}
\hline & \multicolumn{2}{c}{ Readmission } & \multicolumn{2}{c}{ Late AF } & \multicolumn{2}{c}{ All-Cause Mortality } \\
\cline { 2 - 7 } & Univariable & Multivariable & Univariable & Multivariable $^{\mathrm{b}}$ & Univariable $^{\text {Multivariable }}$ \\
\cline { 2 - 7 } & HR $(95 \% \mathrm{CI})$ & HR $(95 \% \mathrm{CI})$ & HR $(95 \% \mathrm{CI})$ & HR (95\% CI) & HR (95\% CI) & HR (95\% CI) \\
\hline POAF & $1.08(0.77 ; 1.51)$ & $0.98(0.69 ; 1.39)$ & $4.80(1.74 ; 13.24)$ & $5.03(1.80 ; 14.10)$ & $1.05(0.65 ; 1.70)$ & $0.95(0.58 ; 1.55)$ \\
\hline
\end{tabular}

${ }^{a}$ Adjusted for age, sex and hypertension, ${ }^{\mathrm{b}}$ Adjusted for hypertension, $\mathrm{POAF}=$ postoperative atrial fibrillation, Late AF = Atrial fibrillation developing after 30 postoperative days.

(a)

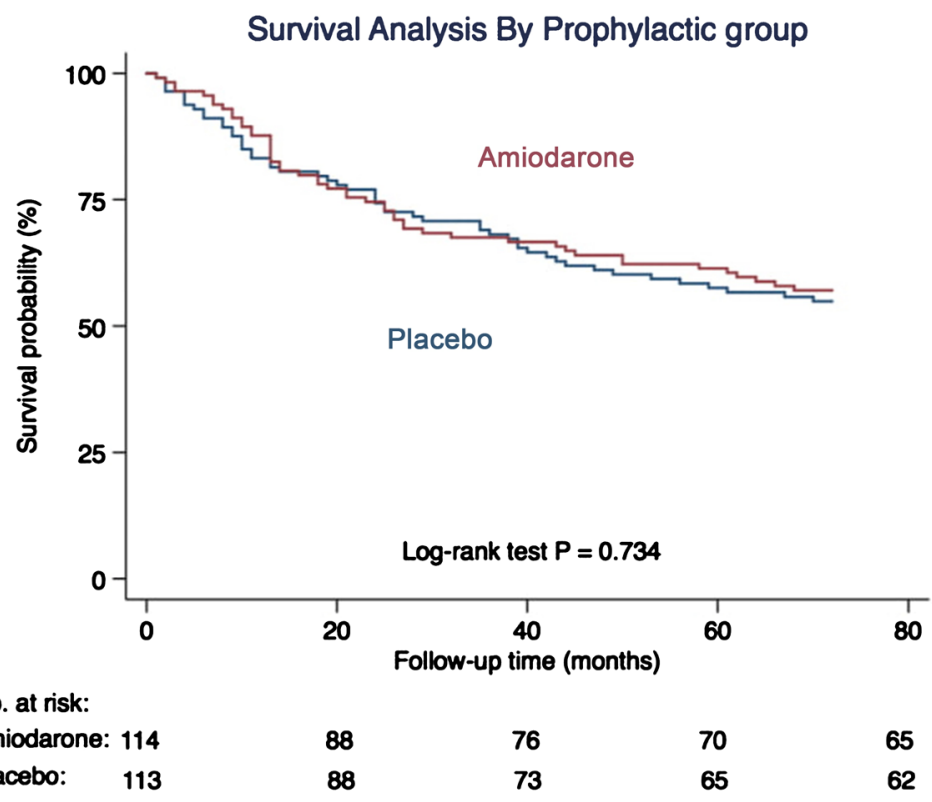

(b)

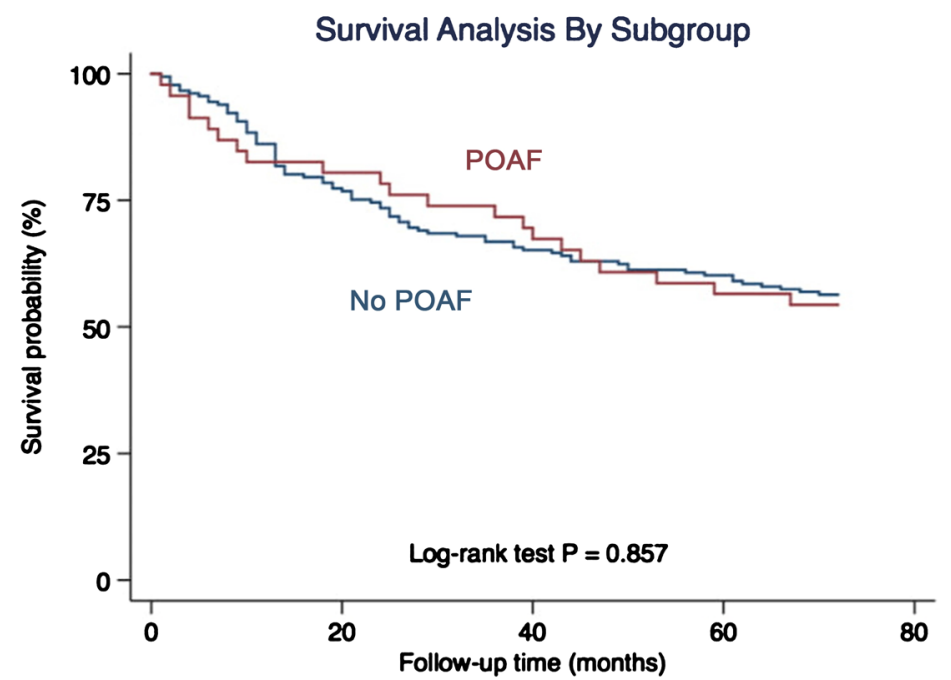

No. at risk:

POAF:

No POAF: 181
37

139
31

118
26

109
25

102

Figure 2. Kaplan Meier survival curves displaying long-term survival of patients undergoing surgery for lung cancer, stratified by treatment groups (a), and subgroups (b). POAF indicates patient with POAF; No POAF, patient without POAF. Risk tables are displayed under the curves. 
was more than $50 \%(56 \%)$ at the last point, and therefore, a median survival time could not be computed. However, we were able to compare the survival curves and found no difference in the long-term overall survival between the prophylactic groups or the subgroups (Table 3 and Table 4).

\subsection{Competing Risk Analysis}

The cumulative incidens function (CIF) of readmission and late AF with death as a competing risk, stratified by prophylactic groups (A) and subgroups (B), are presented in Figure 3 and Figure 4. We found no statistically significant difference in the risk of readmission, between groups. When comparing subgroups,
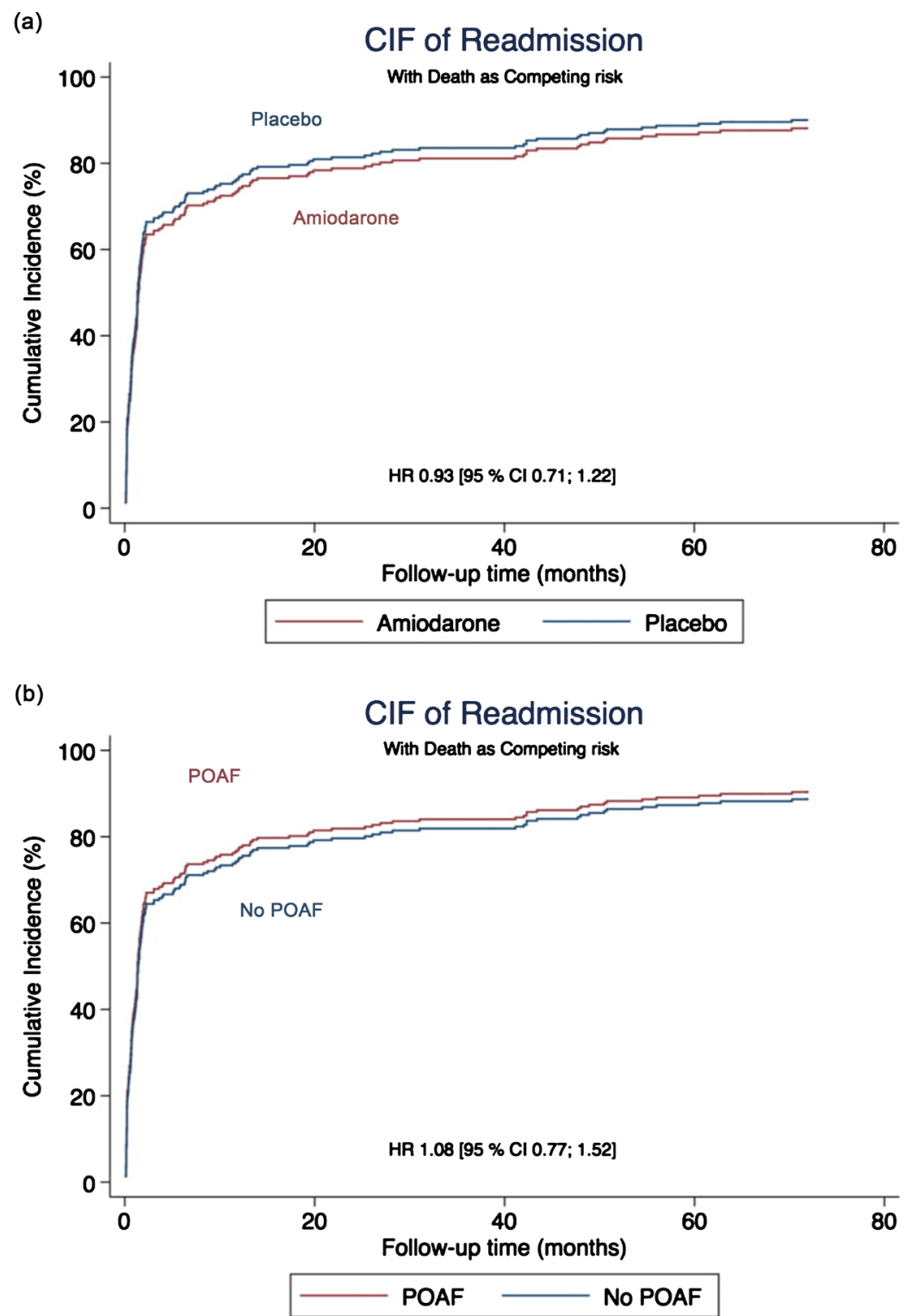

Figure 3. Cumulative incidence function (CIF) of readmission, with death as a competing risk, stratified by treatment groups (a), and subgroups (b). Competing risk regression was used to estimate subdistribution HR (SHR). POAF indicates patient with POAF; No POAF, patient without POAF. 
(a)

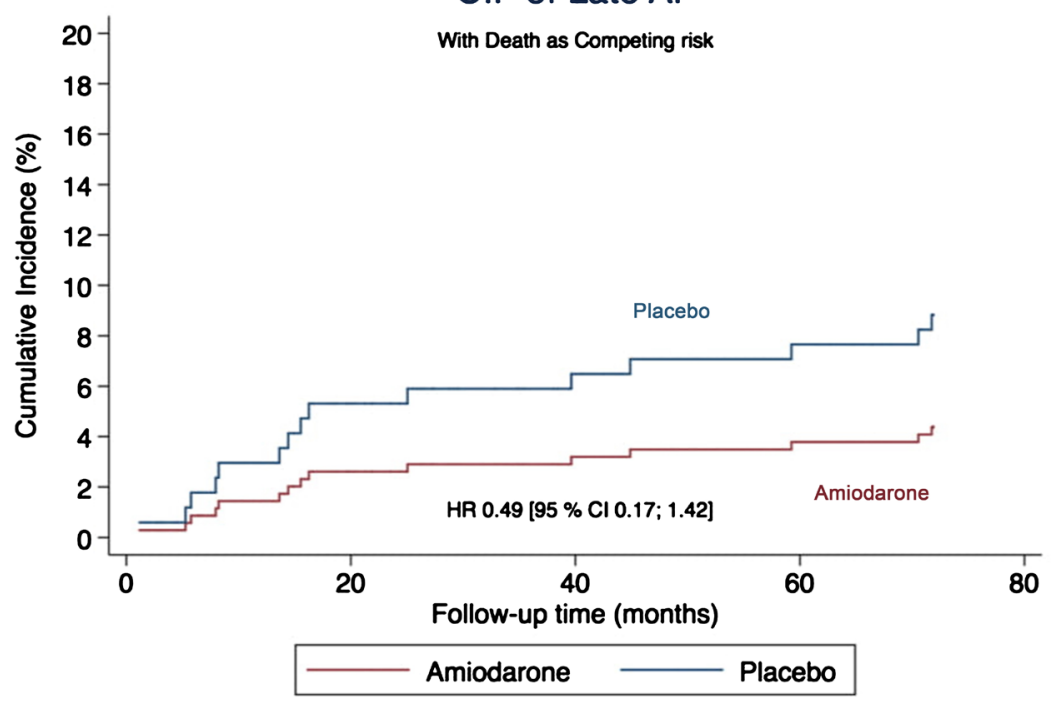

(b)

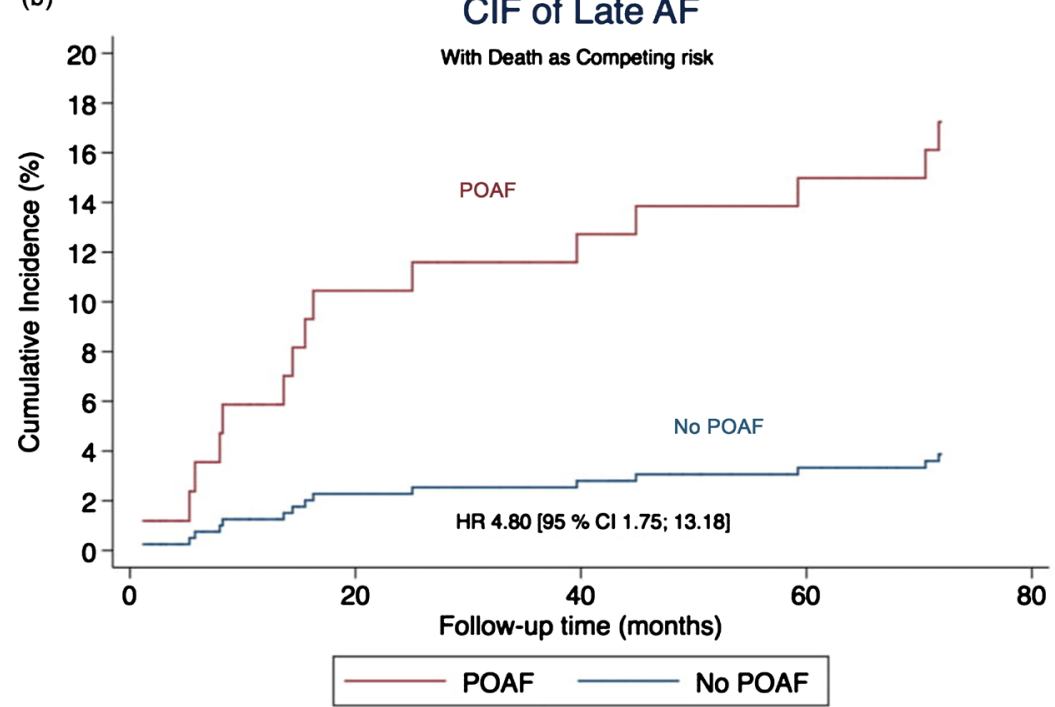

Figure 4. Cumulative incidence function (CIF) of late AF, with death as a competing risk, stratified by treatment groups (a), and subgroups (b). Competing risk regression was used to estimate subdistribution HR (SHR). POAF indicates patient with POAF; No POAF, patient without POAF.

we found POAF to be associated with an increased risk of late AF, when accounting for death as a competing risk. We found no statistically significant difference in the risk of late $\mathrm{AF}$, between prophylactic groups.

\section{Discussion}

In this 6-year follow-up study, we investigated the long-term effect of prophylactic amiodarone, in patients undergoing surgery for lung cancer.

When evaluating long-term side effects of briefly admitted amiodarone in high dose for five days as a prophylactic agent for atrial fibrillation, we found a statistically significant increased risk of head and neck cancer and pleural 
empyema within the placebo group, which was most likely due to chance. Both diagnoses are furthermore known related to the primary lung cancer disease.

Therefore, we did not find any contra-indications in long-time side-effects for using amiodarone in a short period as a high dose prophylactic agent against atrial fibrillation, since the long-term overall survival was not statistically significant different within prophylactic groups.

The use of amiodarone has previously been associated with a number of short-term side effects and more serious adverse effects including skin cancer and toxicity of lung, thyroid and liver [18] [19] [20] [21] [22]. In this current study, we found no statistically significant increased risk for patients who had amiodarone prophylaxis in the PASCART regime.

Further, we investigated the long-term prognosis of patients developing POAF. Patients developing POAF had an approximately a 5-time increased risk of late AF but not an increased risk of readmission and long-term all-cause mortality with-in 7 years of follow-up. These findings suggest that we in Denmark have a good health care system, which detects atrial fibrillation and insures that the patients developing AF are detected and well threatened. Though, it might be due to inadequate power when adjusting for possible confounders.

\section{Strength and Limitations}

The main strengths of our study are the population, which were included from a double-blinded, randomized control trial, and the data source, as follow-up data were obtained from validated national databases. Additionally, the original PASCART study was powered by the number of patients included, reflecting a verified sample size calculation, minimizing type II error. Naturally, some limitations should be noted. First, the study was based on data from a single centre study, which may limit the generalizability of the results, though, the surgical procedures were performed by 6 different thoracic surgeons. Second, some of the follow-up data were based on diagnostic codes and the validity of the results, therefore, depends on similar reporting. Third, like many other retrospective studies, our study contained missing data. In total, missing data were present in 20 baseline variables, with missing values ranging from $0.2 \%$ to $11.8 \%$.

\section{Conclusion}

Amiodarone was found to be a safe option in the prophylactic setting of PASCART within a 6-year follow-up period and should be considered in patients undergoing lobectomy for lung-cancer. POAF was found to increase late AF by a factor 5 .

\section{Acknowledgements and Disclosure}

This study was conducted with external government funding regarding salary and other costs. No author has any conflict of interest. 


\section{Conflicts of Interest}

The authors declare no conflicts of interest regarding the publication of this paper.

\section{References}

[1] Bailey, C.C. and Betts, R.H. (1943) Cardiac Arrhythmias Following Pneumonectomy. New England Journal of Medicine, 229, 356-359. https://doi.org/10.1056/NEJM194308262290902

[2] Cheng, W.L., Kao, Y.H., Chen, S.A. and Chen, Y.J. (2016) Pathophysiology of Cancer Therapy-Provoked Atrial Fibrillation. International Journal of Cardiology, 219, 186-194. https://doi.org/10.1016/j.ijcard.2016.06.009

[3] Iwata, T., Nagato, K., Nakajima, T., Suzuki, H., Yoshida, S. and Yoshino, I. (2016) Risk Factors Predictive of Atrial Fibrillation after Lung Cancer Surgery. Surgery Today, 46, 877-886. https://doi.org/10.1007/s00595-015-1258-4

[4] Farmakis, D., Parissis, J. and Filippatos, G. (2014) Insights into Onco-Cardiology: Atrial Fibrillation in Cancer. Journal of the American College of Cardiology, 63, 945-953. https://doi.org/10.1016/j.jacc.2013.11.026

[5] Ueda, T., Suzuki, K., Matsunaga, T., Takamochi, K. and Oh, S. (2018) Postoperative Atrial Fibrillation Is Less Frequent in Pulmonary Segmentectomy Compared with Lobectomy. General Thoracic and Cardiovascular Surgery, 66, 95-100.

https://doi.org/10.1007/s11748-017-0858-x

[6] Kavurmaci, O., Akcam, T.I., Ergonul, A.G., Turhan, K., Cakan, A. and Cagirici, U. (2017) Is the Risk of Postoperative Atrial Fibrillation Predictable in Patients Undergoing Surgery Due to Primary Lung Cancer? Heart, Lung and Circulation, 27, 835-841. https://doi.org/10.1016/j.hlc.2017.06.729

[7] Kolokotroni, S.M., Toufektzian, L., Harling, L. and Bille, A. (2017) In Patients Undergoing Lung Resection Is It Safe to Administer Amiodarone Either as Prophylaxis or Treatment of Atrial Fibrillation? Interactive CardioVascular and Thoracic Surgery, 24, 783-788. https://doi.org/10.1093/icvts/ivx007

[8] Patel, A.J. and Hunt, I. (2014) Review of the Evidence Supports Role for Routine Prophylaxis against Postoperative Supraventricular Arrhythmia in Patients Undergoing Pulmonary Resection. Interactive Cardio Vascular and Thoracic Surgery, 19, 111-116. https://doi.org/10.1093/icvts/ivu082

[9] Zhang, L. and Gao, S. (2016) Systematic Review and Meta-Analysis of Atrial Fibrillation Prophylaxis after Lung Surgery. Journal of Cardiovascular Pharmacology, 67, 351-357. https://doi.org/10.1097/FJC.0000000000000351

[10] Riber, L.P., Larsen, T.B. and Christensen, T.D. (2014) Postoperative Atrial Fibrillation Prophylaxis after Lung Surgery: Systematic Review and Meta-Analysis. The Annals of Thoracic Surgery, 98, 1989-1997. https://doi.org/10.1016/j.athoracsur.2014.06.069

[11] Zhao, B.C., Huang, T.Y., Deng, Q.W., Liu, W.F., Liu, J., Deng, W.T., et al. (2017) Prophylaxis against Atrial Fibrillation after General Thoracic Surgery: Trial Sequential Analysis and Network Meta-Analysis. Chest, 151, 149-159.

https://doi.org/10.1016/j.chest.2016.08.1476

[12] Riber, L.P., Christensen, T.D., Jensen, H.K., Hoejsgaard, A. and Pilegaard, H.K. (2012) Amiodarone Significantly Decreases Atrial Fibrillation in Patients Undergoing Surgery for Lung Cancer. The Annals of Thoracic Surgery, 94, 339-346.

https://doi.org/10.1016/j.athoracsur.2011.12.096 
[13] Kirchhof, P., Benussi, S., Kotecha, D., Ahlsson, A., Atar, D., Casadei, B., et al. (2016) 2016 ESC Guidelines for the Management of Atrial Fibrillation Developed in Collaboration with EACTS. European Journal of Cardio-Thoracic Surgery, 50, 2893-2962. https://doi.org/10.1093/ejcts/ezw313

[14] Schmidt, M., Maeng, M., Jakobsen, C.J., Madsen, M., Thuesen, L., Nielsen, P.H., et al. (2010) Existing Data Sources for Clinical Epidemiology: The Western Denmark Heart Registry. Clinical Epidemiology, 2, 137-144. https://doi.org/10.2147/CLEP.S10190

[15] Lynge, E., Sandegaard, J.L. and Rebolj, M. (2011) The Danish National Patient Register. Scandinavian Journal of Public Health, 39, 30-33. https://doi.org/10.1177/1403494811401482

[16] Helweg-Larsen, K. (2011) The Danish Register of Causes of Death. Scandinavian Journal of Public Health, 39, 26-29. https://doi.org/10.1177/1403494811399958

[17] Fine, J. and Gray, R. (1999) A Proportional Hazards Model for the Subdistribution of a Competing Risk. Journal of the American Statistical Association, 94, 496-509. https://doi.org/10.1080/01621459.1999.10474144

[18] Teerakanok, J., Tantrachoti, P., Chariyawong, P. and Nugent, K. (2016) Acute Amiodarone Pulmonary Toxicity after Surgical Procedures. The American Journal of Medical Sciences, 352, 646-651. https://doi.org/10.1016/j.amjms.2016.08.013

[19] Jackevicius, C.A., Tom, A., Essebag, V., Eisenberg, M.J., Rahme, E., Tu, J.V., et al. (2011) Population-Level Incidence and Risk Factors for Pulmonary Toxicity Associated with Amiodarone. The American Journal of Cardiology, 108, 705-710. https://doi.org/10.1016/j.amjcard.2011.04.024

[20] Trohman, R.G., Sharma, P.S., McAninch, E.A. and Bianco, A.C. (2018) Amiodarone and the Thyroid Physiology, Pathophysiology, Diagnosis and Management. Trends in Cardiovascular Medicine, 29, 285-295. https://doi.org/10.1016/j.tcm.2018.09.005

[21] Hashmi, A., Keswani, N.R., Kim, S. and Graham, D.Y. (2016) Hepatic Dysfunction in Patients Receiving Intravenous Amiodarone. The Southern Medical Journal, 109, 83-86.

[22] Maoz, K.B., Dvash, S., Brenner, S. and Brenner, S. (2009) Amiodarone-Induced Skin Pigmentation and Multiple Basal-Cell Carcinomas. International Journal of Dermatology, 48, 1398-1400. https://doi.org/10.1111/j.1365-4632.2008.03819.x 